\title{
In Vitro Modification of Bacterial Cyanophycin and Cyanophycin Dipeptides Using Chemical Agents Towards Novel Variants of the Biopolymer
}

\author{
Lars Wiefel $^{1}$ - Felix Bachmann ${ }^{1}$ - Jeroen Terwort ${ }^{1}$ - Alexander Steinbüchel ${ }^{1,2}$
}

Received: 17 April 2019 / Accepted: 5 July 2019 / Published online: 17 July 2019

(c) The Author(s) 2019

\begin{abstract}
Variations of the composition of cyanophycin (CGP) have been investigated since the early 2000s. Modifications of the polymer are of academical interest, but also expand the number of putative applications for CGP and its dipeptides in fields like food supplementations, and medical and cosmetic applications. Until recently variations of the composition occurred only in vivo. However, in the last years, two procedures using chemical or enzymatic in vitro modification were successfully applied. Since chemical treatments were more effective and reached higher conversion rates, a search for reagents and their applicability to conduct reactions with CGP was done. The reaction of CGP with methyl isocyanate resulted in the conversion of $50 \%$ of the lysine residues, while only $3 \%$ of the arginine residues were modified. However, using digested CGP dipeptides, the conversion rates of lysine increased slightly to $72 \%$, while the conversion of arginine reached $96 \%$. Using formaldehyde, CGP could be methylated with a conversion rate of $84 \%$ for lysine and $15 \%$ for arginine. Acetylation of lysine residues was obtained using acetic anhydride, reaching a conversion rate of $100 \%$ for a single acetylation, where $63 \%$ of the residues were acetylated twice. Arginine residues could be acetylated at a rate of $89 \%$. Diacetyl could be added to $80 \%$ of all arginine residues, while lysine was not targeted by the compound. Other agents were also tested, but showed lesser or no conversion and/or inconclusive results. Overall, the tested reactions confirm the viability of chemical CGP modification for future approaches.
\end{abstract}

Keywords Cyanophycin $\cdot$ Chemical modification $\cdot$ Arginine $\cdot$ Lysine $\cdot$ In vitro

\section{Introduction}

The polyamide cyanophycin (CGP) has been in the focus of various studies over the last few decades and especially since the early 2000s. The main focus of these studies was usually the identification of novel production strains or the optimization of the production of CGP in general. Modifications of the CGP composition were mostly out of the scope of academic interest and were considered as a characteristic of the strain or the CGP-producing cyanophycin synthetase.

Alexander Steinbüchel

steinbu@uni-muenster.de

1 Institut für Molekulare Mikrobiologie Und Biotechnologie , Westfälische Wilhelms-Universität Münster, Corrensstraße 3, 48149 Münster, Germany

2 Environmental Science Department, King Abdulaziz University , Jeddah, Saudi Arabia
For example, examining the characteristics of these variations yielded interesting insights in the solubility behavior of the polymer (Wiefel and Steinbüchel 2014). Aside from academic interest, CGP as well as CGP-derived dipeptides also have several putative applications in areas like food supplementations or medical and cosmetic applications were an increased variety in the composition can expand the range of potential uses (Sallam and Steinbüchel 2010). Therefore, actively modifying the composition of CGP has become more relevant in recent years. Usually, variations of the composition occurred only in vivo, either naturally as a characteristic of the producing organism, or intended using selectively chosen and modified strains and/or specifically optimized culture conditions. Strains of E. coli are known to incorporate copious amounts of lysine into the CGP, replacing arginine residues in the side-chains (Kroll et al. 2011, Wiefel et al. 2014), while strains of S. cerevisiae and $P$. putida incorporate citrulline, also replacing arginine (Steinle et al. 2009; Wiefel et al. 2011). 
However, in recent years, two novel procedures using chemical or enzymatic in vitro modification of the CGP were successfully applied. The first chemical modification of CGP was reported by Frommeyer et al. (2014) by the guanidinylation of a lysine-rich CGP with O-methylisourea. This reaction completely converted the lysine residues of CGP to homoarginine (Frommeyer et al. 2014). The feasibility of enzymatic CGP modification was demonstrated by Wiefel and Steinbüchel (2016) using a peptidyl arginine deiminase from Oryctolagus cuniculus, which normally introduces post-translational modifications on several proteins, to catalyze the conversion of arginine residues to citrulline. The conversion rate was, however, only $7.5 \mathrm{~mol} \%$ of the CGPs amino acids, likely due to product inhibition by the citrulline.

While both methods successfully introduced modifications, chemical treatments appeared to be more effective and reach higher conversion rates at the targeted amino acid. Hence, a broad search for applicable reactions was conducted and resulted in a set of putative reactions that were subsequently tested. Generating a product with interesting and/or novel applications was desirable but not necessary, since the main goal of this study was to evaluate and test how viable known reactions used to modify amino acids and proteins can be applied to CGP. Hence, the selection was based on several criteria such as the availability, cost, and toxicity of the required reactants, the stability of the product, and, of course, how likely the reaction was going to work with CGP. The search focussed on reactions targeting the arginine and lysine residues of CGP and the corresponding dipeptides (Fig. 1), since the side-chains of CGP synthesized by recombinant $E$. coli primarily consist of these two amino acids and are, therefore, readily available in larger quantities. The selection included reactions with cyanates, the use of pyridoxal phosphate and acetyl phosphate, the methylation of amino groups using formaldehyde and sodium borohydride, acetylation with acetic anhydride, and reactions with diacetyl.

Reaction of lysine with cyanates and their derivatives, such as isothiocyanate, to form the corresponding thiourea via nucleophilic addition are known (Baslé et al. 2010; Jaisson et al. 2006; Lundblad 1994; Stark 1972) and were used for investigations on lipoproteins (Weisgraber et al. 1978), fructose 1,6-bisphosphatase (Hubert et al. 1986), and collagen (Jaisson et al., 2006). Reactions of amino groups with cyanate can take place under neutral $\mathrm{pH}$ conditions (Stark 1972), but, due to the deprotonation of the lysine side chain at higher $\mathrm{pH}$ levels, the reaction of the $\varepsilon$-amino group is subjected to an alkaline pH regime of 9-9.5 (Baslé et al. 2010). Due to the introduction of sulfur to the lysine linkage, opportunities for other moieties are possible (Baslé et al. 2010), making this reaction an interesting modification for CGP to expand upon. Also, a reaction of cyanates with arginine is

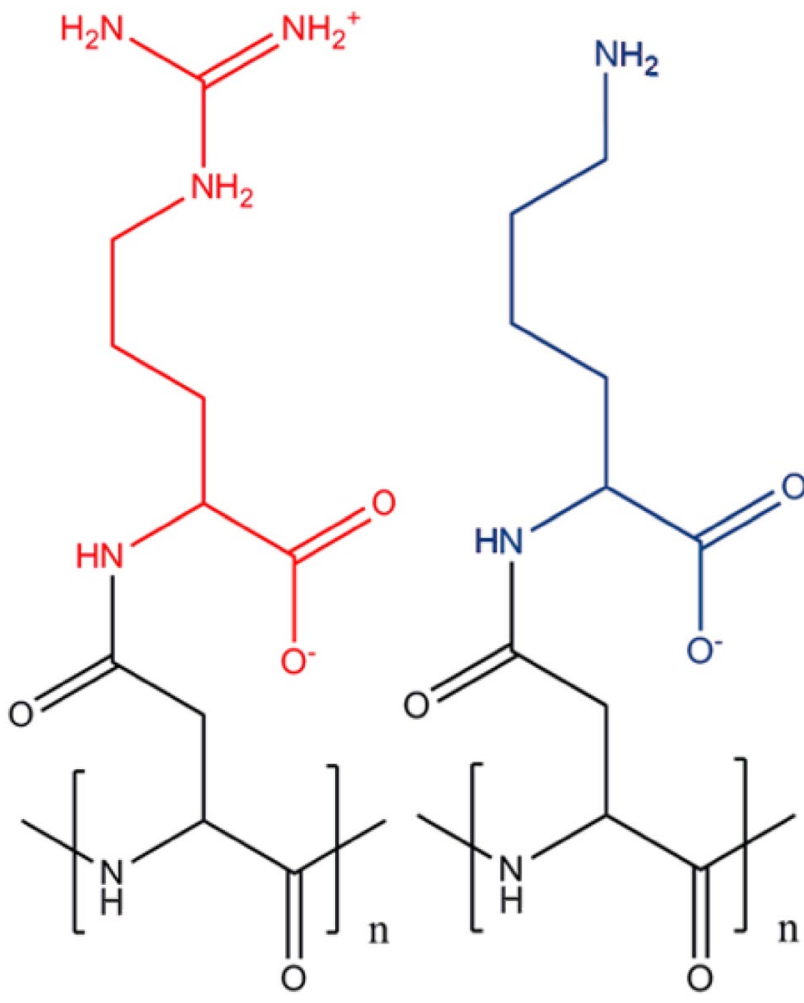

Fig. 1 The two most common dipeptides of the cyanophycin polymer synthesized by $E$. coli, consisting of aspartic acid as part of the backbone plus arginine (left) or of aspartic acid plus lysine (right)

known (Sun et al. 2014). Based on the literature mentioned before, the selected cyanates were methyl isothiocyanate (MITC), guanidine isothiocyanate (GITC), and ammonium isothiocyanate (AITC). The expected products of the reactions are shown in Fig. 2.

Another possible modification is methylation. Amino groups can be methylated with formaldehyde and sodium borohydride $\left(\mathrm{NaBH}_{4}\right)$, where the corresponding reaction product is mainly the di-methylated amino group derivative, since the mono-methylated derivative rapidly reacts further and gets methylated again. The modification is possible in a $\mathrm{pH}$ range from 7 to 10 ; however, below $\mathrm{pH} 9$, $\mathrm{NaBH}_{4}$ becomes instable, while at $\mathrm{pH} 9$ and $0{ }^{\circ} \mathrm{C}$, only the amino groups of proteins are modified (Means 1977; Means and Feeney 1968). $\mathrm{NaCNBH}_{3}$ can be used instead of $\mathrm{NaBH}_{4}$ to increase the efficiency, which also has the advantage that due to the higher stability of $\mathrm{NaCNBH}_{3}$, it is possible to perform the reaction at neutral $\mathrm{pH}$ (Jentoft and Dearborn 1979). Nevertheless, due to safety reasons, only $\mathrm{NaBH}_{4}$ was used in the experiments. At physiological $\mathrm{pH}$, free lysine also forms trimethyl lysine (Trézl et al. 2003). The guanidino group of arginine is not methylated, but hydroxymethylated with formaldehyde (Fig. 3). Analogues to lysine, mono-, di-, and trihydroxymethylated arginine are formed. Hydroxymethylated arginine seems to function as a carrier molecule for 

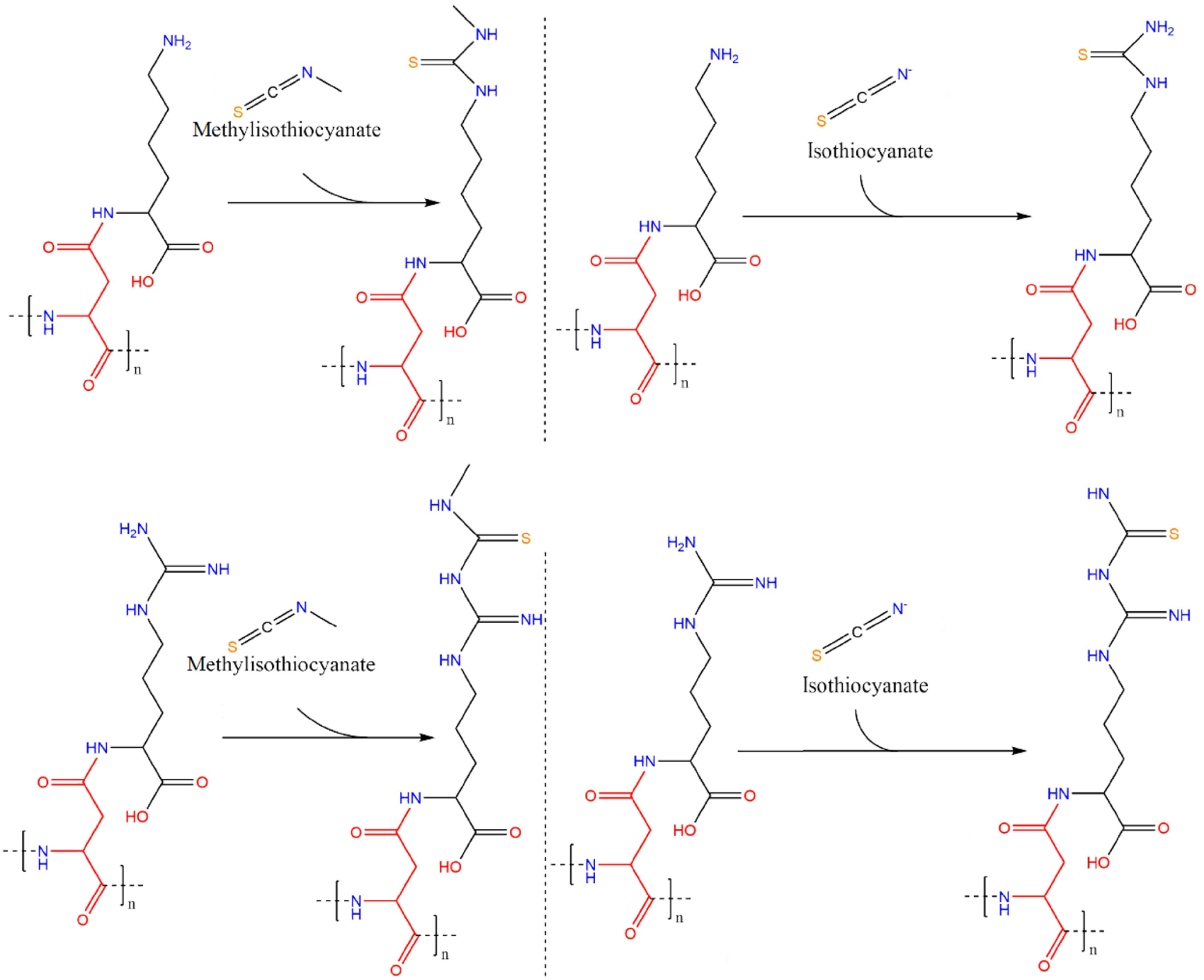

Fig. 2 Proposed cyanophycin derivatives resulting from the reaction with cyanates with the CGP's lysine residue (top) and the arginine residue (bottom). Left reaction of cyanophycin with methyl isothio-

formaldehyde in cells and plays a role in cell signaling and apoptosis (Trézl et al. 2003).

Amino groups can also be acetylated with acetic anhydride, also in proteins and polymers (Fraenkel-Conrat 1957). Treatment of poly-L-lysine with acetic anhydride gave 100\% acetylation of the amino groups (Riordan et al. 1965). There are different possible reaction products for arginine residues, depending on how many and which amino groups of the guanidino group (which has a mesomeric effect) will be acetylated. Reaction of monoalkylguanidines with acetic anhydride gives diacetyl derivatives (Greenhalgh and Bannard 1961), so it is plausible that the guanidino group of arginine can be di- or even triacetylated by acetic anhydride. The product in case that only the $\varepsilon$-amino group of arginine will be acetylated is $N$-acetylarginine; the analogous reaction with lysine will give $N$-acetyllysine. The reaction is shown cyanate, right reaction with ammonium- or guanidine isothiocyanate. Shown is only the isothiocyanate anion; the corresponding cation of the salt and the counterions in $\mathrm{H}_{2} \mathrm{O}$ are not shown

in Fig. 4. Ketene also acetylates amino groups in a broad $\mathrm{pH}$ range from alkaline to $\mathrm{pH}$ 5.0. It also acetylates thiol groups, but with lower reactivity (Herriott 1947). $\mathrm{N}$-acetylimidazole can be used for acetylation of proteins, but has a lower specificity and reactivity for amino groups, and it is mainly used for acetylation of tyrosine residues (Pal et al. 1999; Riordan et al. 1965; Scherer et al. 1992; Zhang et al. 2005). Acetylation of compounds can be interesting for various reasons. For example, acetylation yields a commonly used protection group in organic chemistry (Kociensky 2005) and acetylation of drugs can increase their transport across the blood-brain barrier (Pardridge 2012).

Pyridoxal phosphate (PLP), also known as activated vitamin B6, is a coenzyme involved in many transamination reactions and thus able to bind to amino acids, forming a Schiff base (Miles 1986). In nature, PLP reacts with 


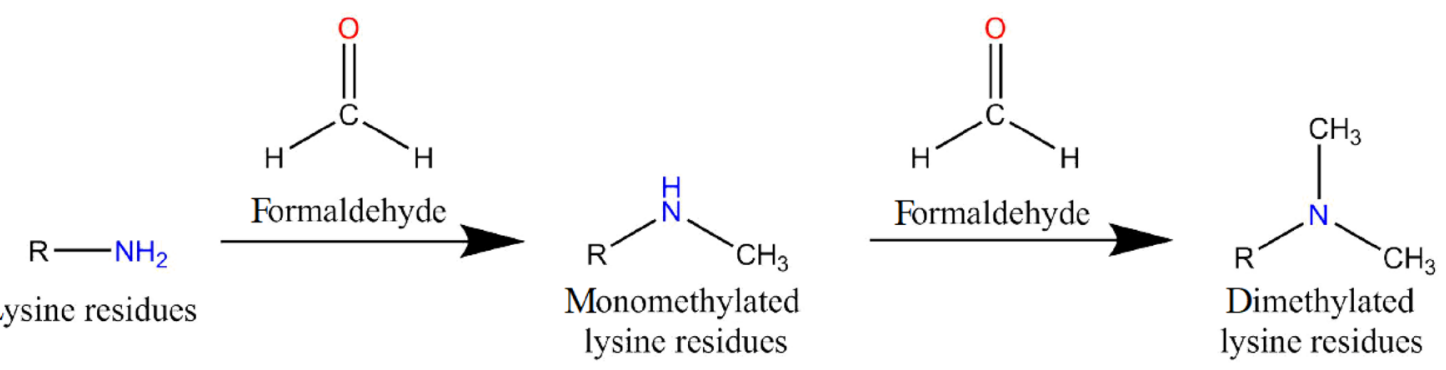

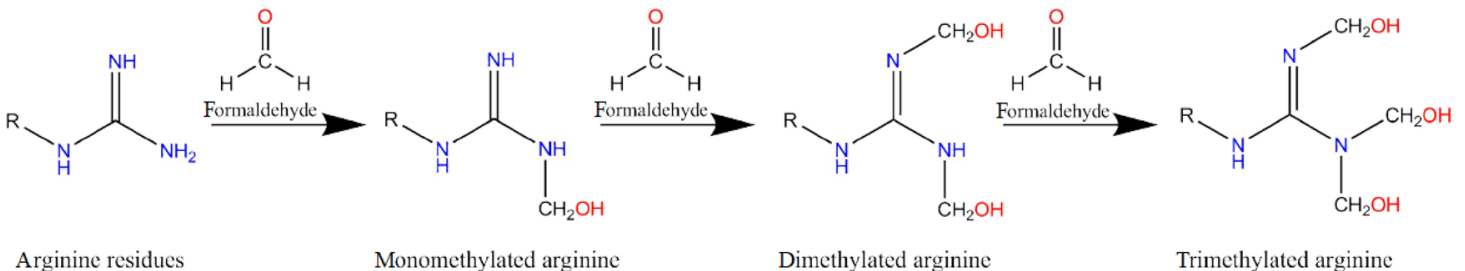

Fig. 3 Putative reaction of lysine and arginine residues with formaldehyde as well as formation of double- or triple-methylated compounds

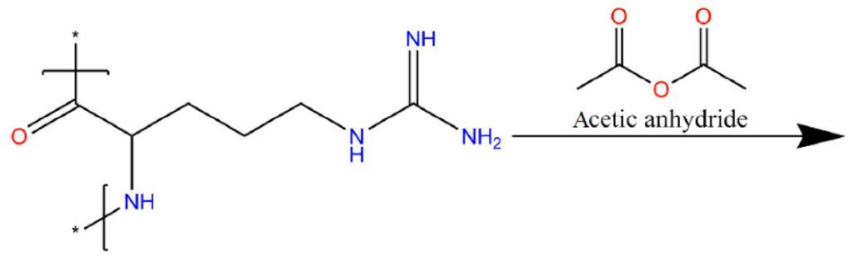<smiles>CC(=O)NC(=N)NCCCC(NC(C)(C)C)C(C)(C)C</smiles>

Arginine<smiles>CC(C)(C)NC(CCCCN)C(=O)C(C)(C)C</smiles>

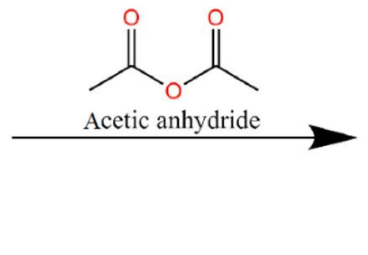<smiles>CC(=O)NCCCCC(NC(C)(C)C)C(=O)O</smiles>

Fig. 4 Putative reaction of lysine and arginine residues with acetic anhydride

the primary amino group of the amino acid, but as already mentioned above, the $\varepsilon$-amino moiety of lysine can be forced to react under deprotonation conditions at an alkaline $\mathrm{pH}$ value to form N6-(pyridoxal phosphate)-lysine (see Fig. 5). Sodium borohydride reduces the formed Schiff base which stabilizes the linkage between PLP and amino acid (Lundblad 1994). Masuda et al. (2005) used the modification of lysine with PLP to study the effect of the lysine residues on the sweetness of lysozyme.

Diacetyl, also referred to as dimethylglyoxal or 2,3-butanedione, reacts specifically with the guanidino group of arginine residues. The reaction was first described by Harden and Norris in 1911 (Harden and Norris 1911). Diacetyl can also be dimerized and trimerized to different stable products, which also react with arginine (Cresswell et al. 1961; Yankeelov et al. 1968; Yankeelov 1970). The reaction, which is shown in Fig. 6, occurs at mild conditions of $25^{\circ} \mathrm{C}$ and neutral $\mathrm{pH}$ (Yankeelov 1970). Diacetyl is cheap but toxic; however, serious health risks are mainly due to inhalation of gaseous diacetyl, which will not occur during proper handling. The structure of the product for the reaction of monomeric diacetyl with $\mathrm{N} \alpha$ acetylarginine is known (Mathews et al. 2010), but the ones for the reaction with dimeric or the different trimeric diacetyl forms are unknown. 
Fig. 5 Addition of PLP to the lysine residue of cyanophycin

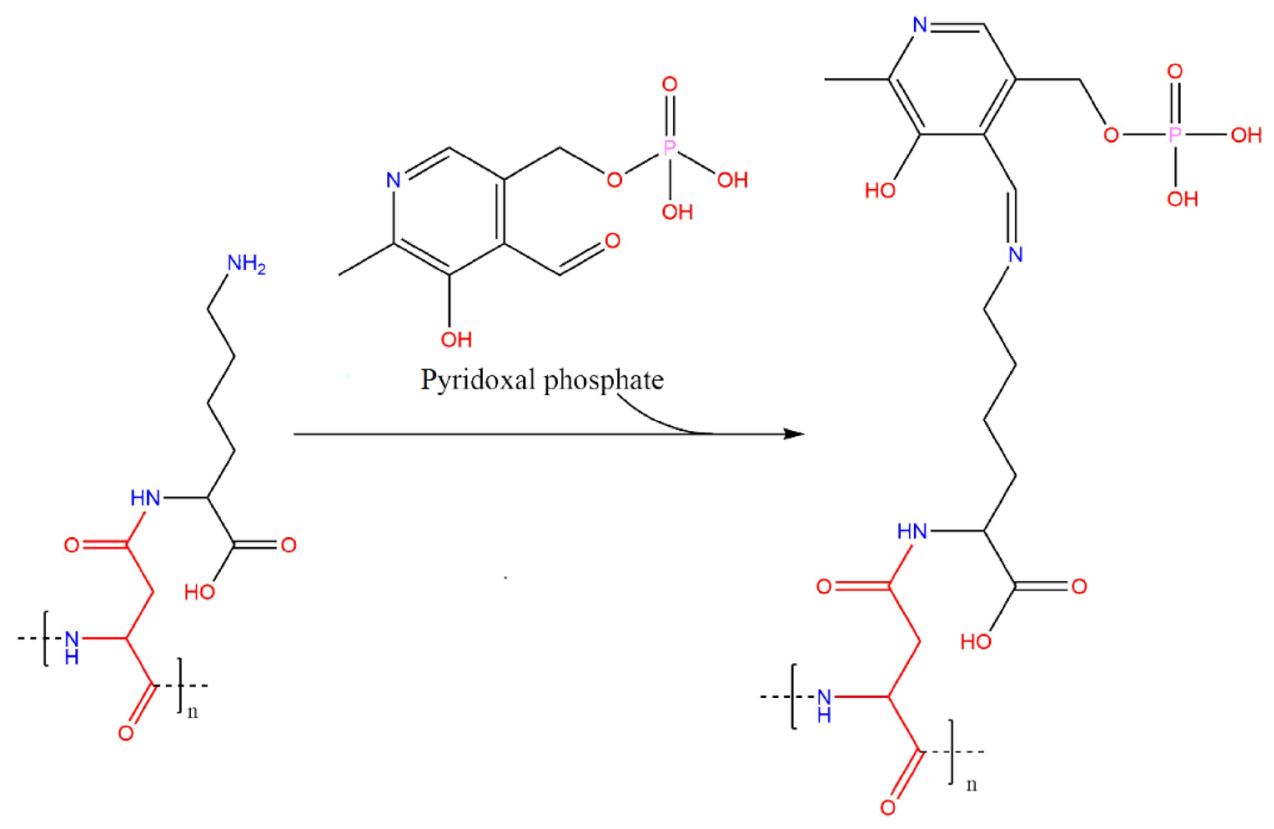

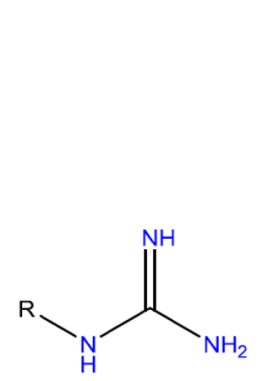

Arginine residues<smiles>CC(=O)C(C)=O</smiles>

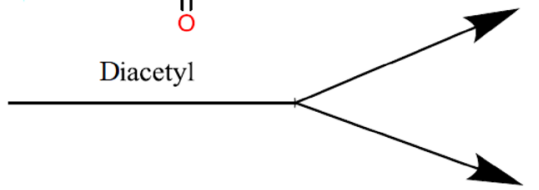<smiles>[2H]NC1=NC(C)(O)C(C)(O)N1</smiles><smiles>CC(=O)C(C)(O)NC(=N)NP</smiles>

\section{Materials and Methods}

\subsection{Synthesis of CGP and Dipeptides}

Escherichia coli HSM174 pCOLADuet::cphA ${ }_{6308}$ (Kroll et al. 2011) was used for CGP production according to Wiefel and Steinbüchel (2014). Within $26 \mathrm{~h}$, a cell density of $13.3 \mathrm{~g} / \mathrm{L}$ was obtained, and an overall CGP content of $34.1 \%$ of the cell dry mass was measured. $67.7 \%$ of the accumulated CGP were insoluble with a lysine content of $15.8 \mathrm{~mol} \%$, while the remaining $32.3 \%$ of the polymer were soluble with a lysine content of $35.8 \mathrm{~mol} \%$.

\subsection{Digestion of CGP}

CGP was also digested for enzyme tests using CGP dipeptides and for analytical purposes. For that, CGP was digested using a purified $\mathrm{CphE}$ (Cyanophycinase, EC 3.4.15.6) from Pseudomonas alcaligenes DIP1 (Sallam et al. 2009) for the generation of a mixture of Asp-Arg and Asp-Lys dipeptides. $10 \mathrm{mg}$ of CGP were mixed with $50 \mu \mathrm{l}$ of $\mathrm{CphE}$ solution $\left(0.8 \mathrm{mg} / \mathrm{ml}\right.$ in $\left.\mathrm{H}_{2} \mathrm{O}\right)$, adjusted to $1 \mathrm{ml}$ with Tris/HCl-buffer (20 mM, pH 8.0), and incubated at $50{ }^{\circ} \mathrm{C}$ for $5 \mathrm{~h}$. Afterwards, the reaction mixture was passed through an ultrafiltration column $(10 \mathrm{kDa}$ exclusion 
size; Vivaspin, Sartorius, Göttingen) to separate the dipeptides from the $\mathrm{CphE}$ in the supernatant. A control without CGP was carried along to ensure that all detected signals derived from the digested CGP. CGP modified in reactions was first separated from the reaction mixture by an ultrafiltration column (10 $\mathrm{kDa}$ exclusion size) which retained the CGP, before being rebuffered with Tris/ $\mathrm{HCl}(20 \mathrm{mM}, \mathrm{pH}$ 8.0), and mixed with CphE. CphE solution was provided by Cysal GmbH, Münster, Germany.

\subsection{Reaction with Cyanates}

Reactions with cyanates were performed with a modified protocol of Weisgraber et al. (1978). A solution of $1 \mathrm{mg}$ per ml CGP was mixed with $20 \mathrm{mg}$ of cyanate per $\mathrm{mg}$ of CGP. Due to the use of mass spectrometric (MS) methods, sodium borate buffer was replaced with $\mathrm{H}_{2} \mathrm{O}_{\text {dest }}$ for GITC and AITC, while the MITC solution also contained 1/4 (v/v) of methanol to solve the cyanate completely. The methanol content was adjusted to the same level after the reaction was completed for analytic purposes. The $\mathrm{pH}$ was adjusted by a $2.5 \%(\mathrm{w} / \mathrm{v})$ ammonia solution. The reaction solution was incubated for $2 \mathrm{~h}$ at $30^{\circ} \mathrm{C}$ and stopped by cooling on ice, dilution with methanol and water, and lowering the $\mathrm{pH}$ to acid levels for MS analysis. The $\mathrm{pH}$ dependence of the reaction was studied in a range from $\mathrm{pH} 8$ to $\mathrm{pH} 10$.

\subsection{Reaction with Formaldehyde}

The reactions with formaldehyde were performed using a modified protocol of Means and Feeney (1968). $100 \mu \mathrm{l}$ of a CGP or CGP dipeptide solutions $(50 \mathrm{mg} / \mathrm{ml})$ was mixed with $900 \mu$ l borate buffer $(50 \mathrm{mM}, \mathrm{pH} 8.2)$. The reaction was started by adding $20 \mu \mathrm{l}$ of $37 \%(\mathrm{v} / \mathrm{v})$ formaldehyde, adding additional $20 \mu \mathrm{l}$ every $10 \mathrm{~min}$ over the next $50 \mathrm{~min}$ for a total volume of $120 \mu \mathrm{l}$. The reaction was performed on ice for $18 \mathrm{~h}$. Afterwards, not digested CGP was digested as previously mentioned, while dipeptides were directly prepared for MS analysis.

\subsection{Reaction with Acetic Anhydride}

Acetylation with acetic anhydride was performed after a modified protocol of Riordan et al. (1965) using $100 \mu \mathrm{l}$ of a CGP or CGP dipeptide solutions $(50 \mathrm{mg} / \mathrm{ml})$, and mixed with $900 \mu \mathrm{l} \mathrm{NaOAc}$ buffer $(40 \mathrm{mM}, \mathrm{pH}$ 7.2). The reaction was started by adding $20 \mu \mathrm{l}$ of acetic anhydride and adding additional $20 \mu \mathrm{l}$ every $10 \mathrm{~min}$ over the next $50 \mathrm{~min}$ for a total volume of $120 \mu \mathrm{l}$. The reaction was performed on ice for $18 \mathrm{~h}$. Afterwards, undigested CGP was digested as previously mentioned, while dipeptides were directly prepared for MS analysis.

\subsection{Reaction with Pyridoxal Phosphate}

The reaction protocol for the reaction of amino acids with pyridoxal phosphate was carried out according to the report of Shapiro et al. (1968). Since CGP and amino acids do not have to be buffered, the use of the sodium phosphate was replaced with $\mathrm{H}_{2} \mathrm{O}_{\text {dest }}$. This also allows the direct measurement of the resulting product in the mass spectrometer. The reaction was set up with $1 \mathrm{mg} / \mathrm{ml}$ of CGP and $1 \mathrm{mM}$ pyridoxal phosphate at a $\mathrm{pH}$ of 8 . The reaction time was $1 \mathrm{~h}$ at $30{ }^{\circ} \mathrm{C}$. The stabilization of the resulting Schiff base by sodium borohydride was only performed with not digested CGP to enable the removal of sodium borohydride by ultrafiltration. For this, the reaction with PLP was started as described above. After $30 \mathrm{~min}$, the sodium borohydride was added up to a concentration of $0.6 \mathrm{mg} / \mathrm{ml}$, leading to a discoloring of the yellow pyridoxal phosphate solution.

\subsection{Reactions with Diacetyl and Diacetyl trimer}

The reactions were performed using $100 \mu \mathrm{l} \mathrm{CGP} \mathrm{or} \mathrm{CGP}$ dipeptide solutions $(50 \mathrm{mg} / \mathrm{ml}), 900 \mu \mathrm{H}_{2} \mathrm{O}$, and $20 \mu \mathrm{l}$ diacetyl. The reaction was performed on ice for $18 \mathrm{~h}$. In case of the diacetyl trimer, $200 \mu \mathrm{l}$ were used in the reaction mixture. The diacetyl trimer was prepared by mixing $20 \mathrm{~mL}$ borate buffer $(50 \mathrm{mM}, \mathrm{pH} 8.2)$ with $3.75 \mathrm{~mL}$ diacetyl, adjust to $\mathrm{pH} 9$ using $\mathrm{NaOH}$, and adjusting the volume to $25 \mathrm{~mL}$ with $\mathrm{H}_{2}$ Odest and stirring for $1 \mathrm{~h}$.

\subsection{Mass Spectrometry (MS)}

Samples were analyzed by MS (LXQ Mass Spectrometer, Finnigan) with LQXTunePlus software (Finnigan). Samples were prepared for analysis by addition of $1 / 3$ of the sample volume of methanol. The production of positive charged ions was supported by adding $0.1 \%$ formic acid, yielding a $\mathrm{pH}$ value in a slightly acidic range of $\mathrm{pH} 6-4$. The $\mathrm{pH}$ value was tested with $\mathrm{pH}$ paper ( $\mathrm{pH}$ 1-11, Macherey-Nagel), and if needed, more formic acid was added until the $\mathrm{pH}$ range was reached. The solution was directly injected into the ion source of the MS at a flow rate of $10 \mu \mathrm{l} / \mathrm{min}$. The basic parameters during measurement used the following values: a capillary temperature of $300{ }^{\circ} \mathrm{C}$, a sheath gas flow rate of $12 \mathrm{Ls} / \mathrm{h}$, an auxiliary gas flow of $6 \mathrm{Ls} / \mathrm{h}$, and a sweep gas flow of $1 \mathrm{~L} / \mathrm{h}$. A mass range from $m / z=50$ to 1,000 was scanned.

\section{Results}

Since both, high lysine content and solubility of the polymer, were desired for the reactions, the soluble lysine-rich CGP produced by E. coli HSM174 pCOLADuet::cphA 6308 was 
chosen for the experiments. This form offered both lysine and arginine residues as targets for chemical modifications.

\subsection{Reactions with Cyanates}

The reactions of CGP and CGP dipeptides were performed with the cyanates MITC, GITC, and AITC. However, only the reaction with MITC showed a noticeable conversion of the amino acid residues. Both other cyanates gave no noticeable modifications (data not shown).

The MS analysis of CGP dipeptides modified with MITC (Fig. 7) showed two products for lysine. One peak at $m / z=335.4$ can be identified as the addition of MITC to either the $\varepsilon$-amino moiety of the lysine or as the addition to the $\alpha$-amino group of the aspartate of an Asp-Lys dipeptide. The other signal at $m / z=403.5$ can be identified as the corresponding double addition on both groups. The double addition is an indication that the lysine residue in undigested cyanophycin might also be modified by MITC. The Asp-Arg dipeptide also showed a signal at $\mathrm{m} / \mathrm{z}=363.4$ corresponding to the addition of MITC. This product is also present in form of a dehydrated peak at $m / z=345.4$ with about one-fourth of the intensity of the parent signal. In addition, it has to be mentioned that traces of the dehydrated product of the single addition to the Asp-Lys dipeptide were detected $(m / z=317.4)$. However, due to its very low intensity, this peak was neglected in the further considerations. This dehydration also occurred in reactions with free lysine and arginine (data not shown), and it is uncertain if this is a side-effect of the reaction itself or an artifact of MS analysis. The reaction was performed at $\mathrm{pH} 8,9$, and 10 , showing increasing conversion rates for both arginine and lysine under more alkalic conditions. At pH $1072.2 \%$ of the AspLys dipeptides and $96.5 \%$ of the Asp-Arg dipeptides were modified (see Table 1). It is noticeable that by changing the $\mathrm{pH}$ from 8 to 9 both, the single and the double additions of MITC, increased, resulting in less unmodified lysine, while the change from $\mathrm{pH} 9$ to 10 resulted in a higher percentage of the lysine exhibiting the double addition, but did not further reduce the percentage of unmodified lysine.

Carrying out the reaction with undigested CGP proved to be problematic, due to solubility issues. The use of $1 / 3$ methanol, which is needed to solve the MITC, in an alkaline $\mathrm{pH}$ regime forces the CGP to precipitate. Thus, a chemical contact between the cyanate and the CGP is unlikely, and the subsequent analysis of the reaction products showed only $3.2 \%$ of the arginine residues being converted to the expected reaction product. The conversion of lysine at a rate

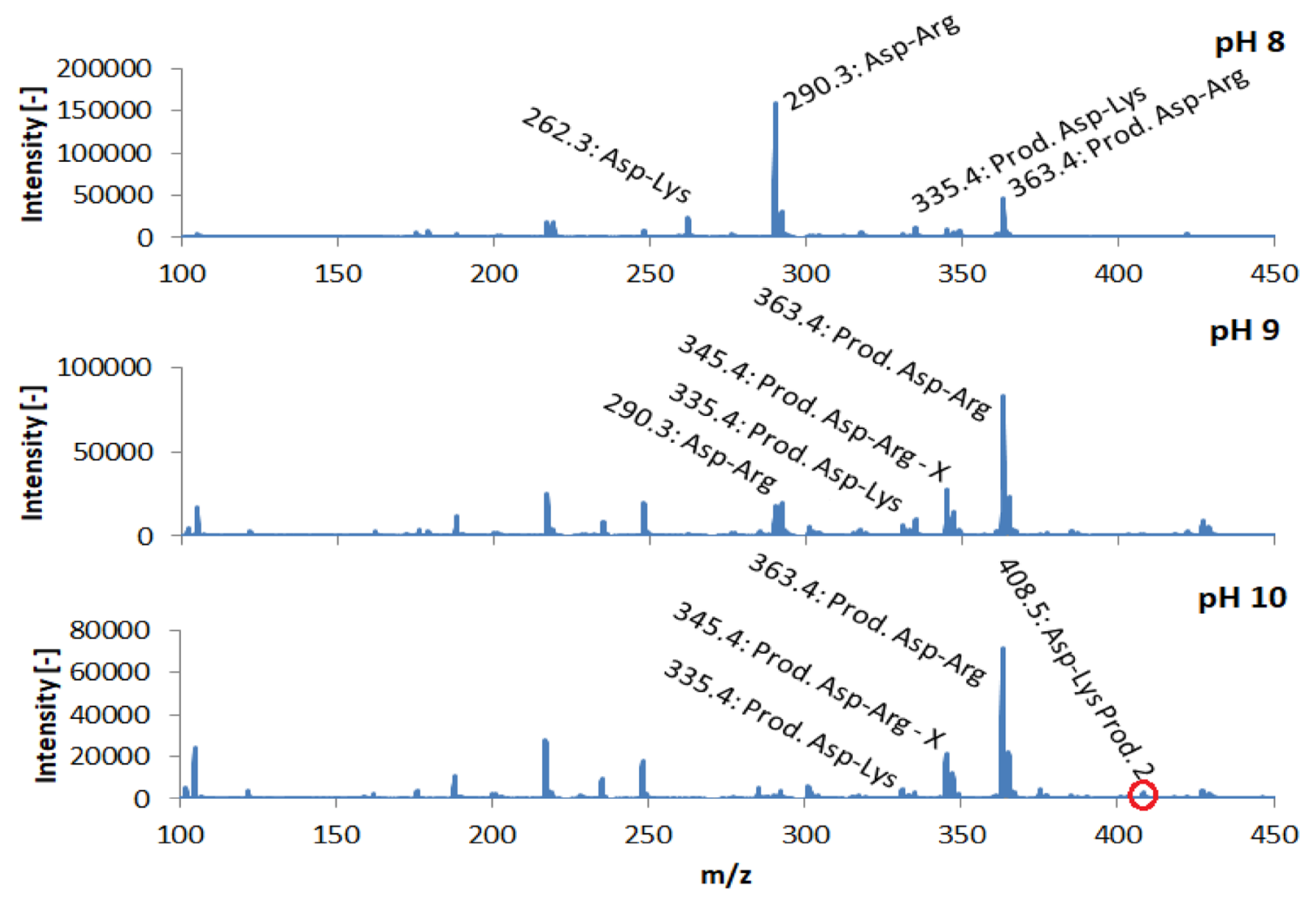

Fig. 7 MS analysis of the reaction of a cyanophycin dipeptide mixture with methyl isothiocyanate (MITC) at $\mathrm{pH} \mathrm{8-10.} \mathrm{The} \mathrm{mass}$ change of Asp-Lys from $\mathrm{m} / \mathrm{z}=262.3$ to 335.4 indicates the addition of one molecule of MITC, and the change to $\mathrm{m} / \mathrm{z}=408.5$ the addition of two molecules. The Asp-Arg mass change from $m / z=290.3$ to 363.4 corresponds to one addition of MITC 
Table 1 Conversion rates of cyanophycin (CGP) reacting with methyl isothiocyanate (MITC)

\begin{tabular}{lllllll}
\hline DP & pH 8 & pH 9 & pH 10 & CGP & pH 8 & pH 10 \\
\hline Asp-Lys 0x & $66.5 \pm 0.3 \%$ & $26.5 \pm 0.3 \%$ & $27.8 \pm 0.1 \%$ & Asp-Lys 0x & $82.4 \pm 0.1 \%$ & $48.5 \pm 0.1 \%$ \\
1x & $31.0 \pm 0.4 \%$ & $59.0 \pm 1.5 \%$ & $35.6 \pm 0.4 \%$ & $1 \mathrm{x}$ & $9.4 \pm 0.7 \%$ & $45.5 \pm 0.0 \%$ \\
$2 \mathrm{x}$ & $2.5 \pm 0.2 \%$ & $14.5 \pm 1.2 \%$ & $36.6 \pm 0.4 \%$ & $2 \mathrm{x}$ & $8.2 \pm 0.4 \%$ & $6.0 \pm 0.1 \%$ \\
Asp-Arg 0x & $75.6 \pm 0.4 \%$ & $17.1 \pm 1.5 \%$ & $3.5 \pm 0.4 \%$ & Asp-Arg 0x & $98.2 \pm 0.0 \%$ & $96.8 \pm 0.7 \%$ \\
$1 \mathrm{x}$ & $24.4 \pm 0.4 \%$ & $82.9 \pm 0.3 \%$ & $96.5 \pm 0.0 \%$ & $1 \mathrm{x}$ & $1.8 \pm 0.1 \%$ & $3.2 \pm 0.1 \%$ \\
\hline
\end{tabular}

CGP and CGP dipeptides (DP) were incubated with MITC at different $\mathrm{pH}$ values and analyzed by mass spectroscopy to determine the conversion rates of Asp-Lys and Asp-Arg dipeptides reacting with MITC

$D P$ reaction performed with CGP dipeptides, $C G P$ reaction performed with undigested CGP, $O x$ no modification, $1 x$ addition of one molecule of MITC, $2 x$ addition of two molecules of MITC of $50.3 \%$, even though much better compared to arginine, was also noticeably worse to reactions with already digested dipeptides ( $72.2 \%$ conversion). In both cases, the conversion rates were also higher at $\mathrm{pH}=10$ than under less alkalic conditions. A pH of 9 was not tested.

\subsection{Methylation using Formaldehyde}

The MS analysis of cyanophycin cleaved by CphE into dipeptides after reaction with formaldehyde exhibited a dominant signal at $m / z=290.17$, which corresponds to the unmodified Asp-Arg dipeptide, while a secondary signal at $m / z=262.17$ refers to unmodified Asp-Lys. A signal measured at $m / z=276.17$ matches with the expected mass of a methylated Asp-Lys dipeptide. The measured conversion rate for unmodified Asp-Lys to methylated Asp-Lys based on the intensities of the two peaks was calculated at $84.4 \%$. There are three very weak additional signals at $m / z=303.17$, 312.17 , and 332.08 which do not correspond to any predicted product of the reaction. No relevant methylation of the Asp-Arg dipeptide was measured.

The MS analysis of dipeptides modified with formaldehyde again showed the highest peak at $\mathrm{m} / z=290.08$ corresponding to the unmodified Asp-Arg dipeptide (Fig. 8). This analysis also shows two smaller signals at $m / z=304.17$ and $m / z=319.92$ and a stronger third signal at $m / z=334.08$. The peak at $m / z=319.92$ matches with the predicted hydroxymethylated Asp-Arg dipeptide, while the signals at $m / z=304.17$ and $m / z=334.08$ were not expected. Most likely, the signal at $m / z=304.17$ results from the methylation of Asp-Arg at the amino group of aspartic acid and the signal at $m / z=334.08$ corresponds to the hydroxyl-methylated form of this methylated dipeptide. Taking this into account, the conversion rates for Asp-Arg of undigested CGP were as follows: $6.8 \%$ were methylated at the amino-group of Asp $(m / z=304.17), 5.4 \%$ were hydroxymethylated at the guanidino group of $\operatorname{Arg}(m / z=319.92)$, and $15.4 \%$ were both methylated and hydroxymethylated $(\mathrm{m} / \mathrm{z}=334.08)$. There was no conversion of the Asp-Lys dipeptide measured in these reactions.

\subsection{Acetylation with Acetic Anhydride}

The MS analysis of cyanophycin modified with acetic anhydride and cleaved by $\mathrm{CphE}$ into dipeptides exhibited various signals. Aside of signals for the unmodified Asp-Lys
Fig. 8 MS analysis of the methylation of Asp-Arg dipeptides using formaldehyde. The change from Asp-Arg at $m / z=290.08$ to 319.92 corresponds to the expected structure of hydroxymethylated Asp-Arg, while $m / z=304.17$ matches Asp-Arg with a methylated Asp amino group. The signal at $m / z=334.08$ matches the predicted mass of an AspArg dipeptide, methylated at the amino group of Asp and hydroxymethylated at the guanidine group of Arg

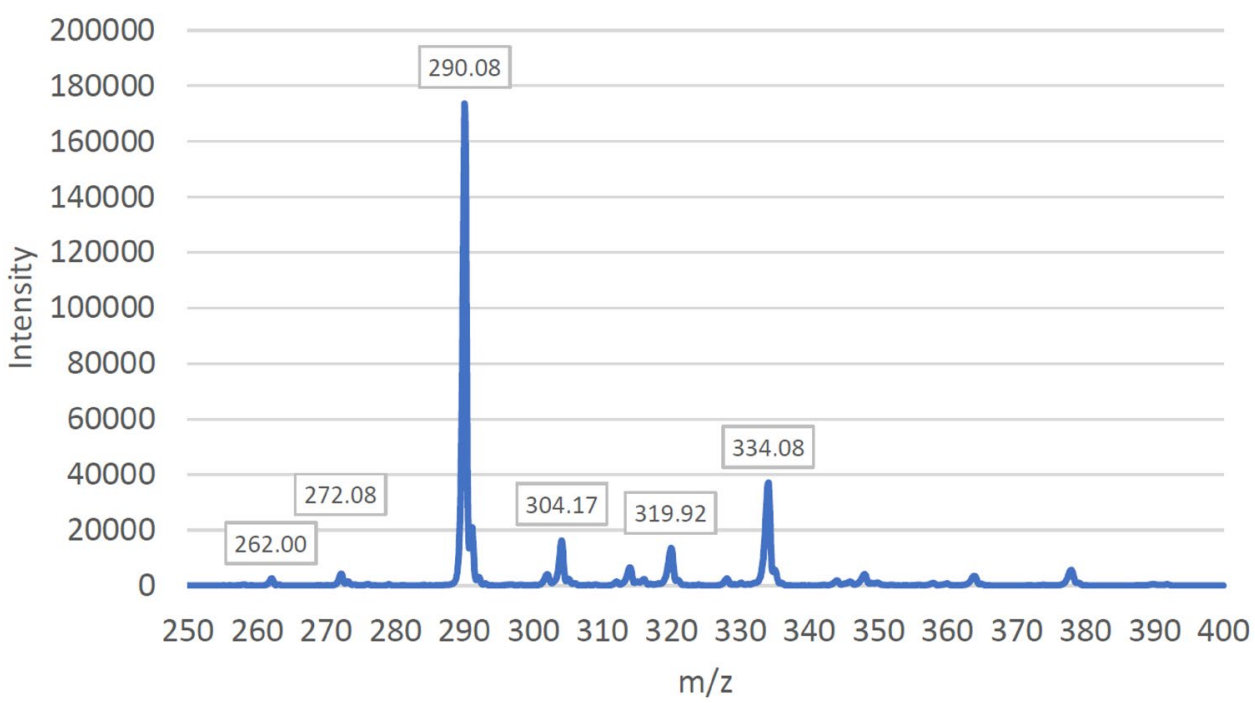


Fig. 9 MS analysis of the reaction of a CGP with acetic anhydride. The mass change of Asp-Lys from $m / z=262.3$ to 304.08 indicates a single acetylation. The Asp-Arg change from $\mathrm{m} / \mathrm{z}=290.0$ to 332.17 corresponds to a single acetylation

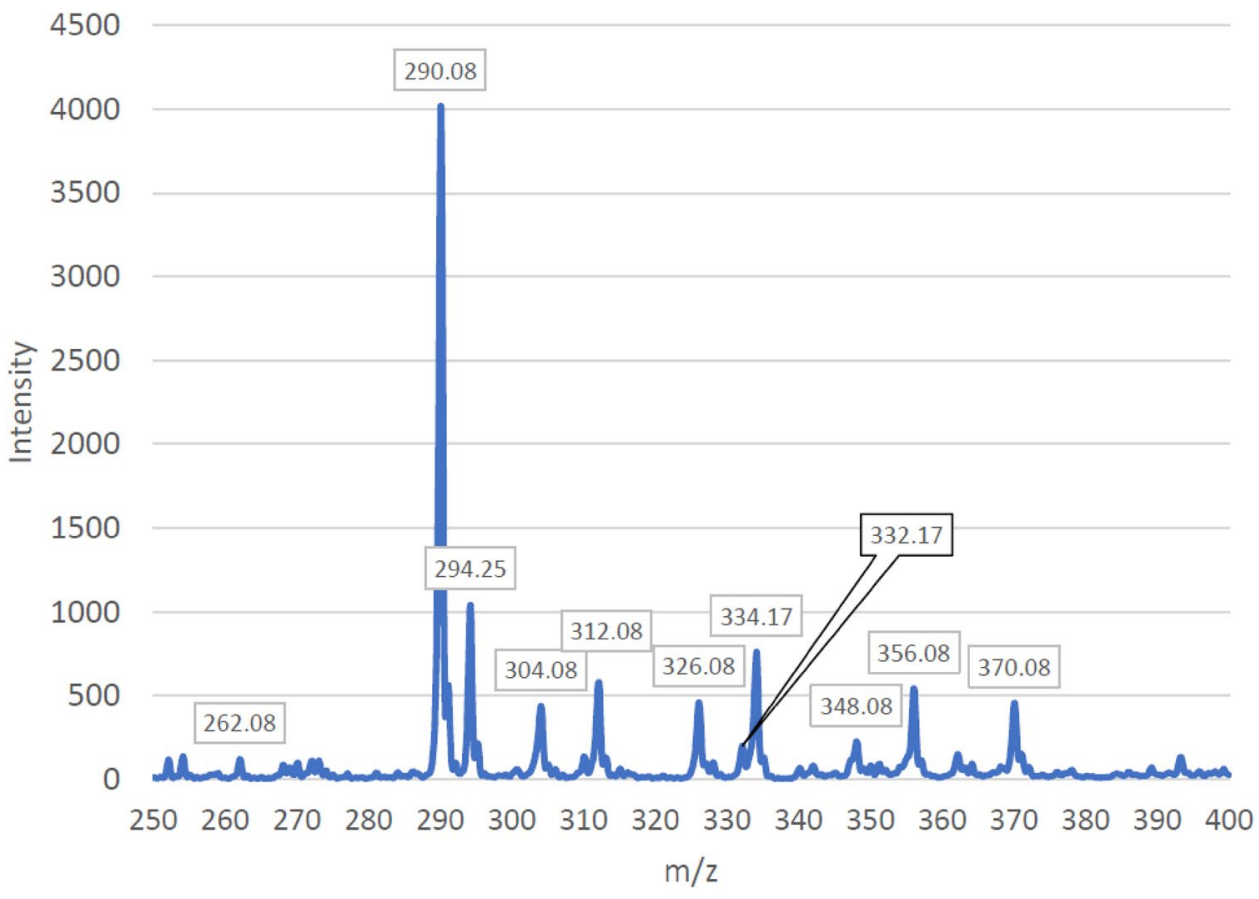

$(m / z=262.08)$ and Asp-Arg $(m / z=290.08)$, signals for the acetylated forms of Asp-Lys $(\mathrm{m} / \mathrm{z}=304.08)$ and Asp-Arg $(\mathrm{m} / z=332.17)$ were detected (Fig. 9). In addition, a series of unexpected signals was detected at $m / z=294.25,312.08$, $326.08,334.17,348.08,356.08$, and 370.08 which allow for various interpretations. The conversion rates of the expected reaction products were calculated as $78.5 \%$ for acetylated Asp-Lys $(\mathrm{m} / z=262.08$ to 304.08$)$ and $4.7 \%$ for acetylated Asp-Arg $(\mathrm{m} / z=290.08$ to 332.17).

The MS analysis of dipeptides modified with acetic anhydride exhibited also the signals for the unmodified dipeptide Asp-Arg at $m / z=290.08$ and the signals for the acetylated products of Asp-Lys and Asp-Arg at $m / z=304.12$ and 331.96 (Fig. 10). The signal for unmodified Asp-Lys at $m / z=262.3$ was only briefly visible during analysis. Since the reaction was performed with already digested CGP, an acetylation of the amino group of the aspartic acid can also occur resulting in a signal matching an Asp-Lys acetylated at the lysine. In addition, a double-acetylated reaction product of Asp-Lys can occur. A signal corresponding to the predicted mass of a double-acetylated Asp-Lys was detected at $m / z=346.00$. A signal at $m / z=314.08$ is likely caused by acetylation of an unknown molecule measured at $m / z=272.2$, which also appeared in other dipeptide reaction
Fig. 10 MS analysis of the reaction of a CGP dipeptide mixture with acetic anhydride. The mass change of Asp-Lys from $m / z=262.3$ (not visible) to 304.12 indicates a single acetylation, while $\mathrm{m} / \mathrm{z}=346.0$ indicates a double acetylation. The Asp-Arg change from $\mathrm{m} / \mathrm{z}=290.0$ to 331.96 corresponds to a single acetylation

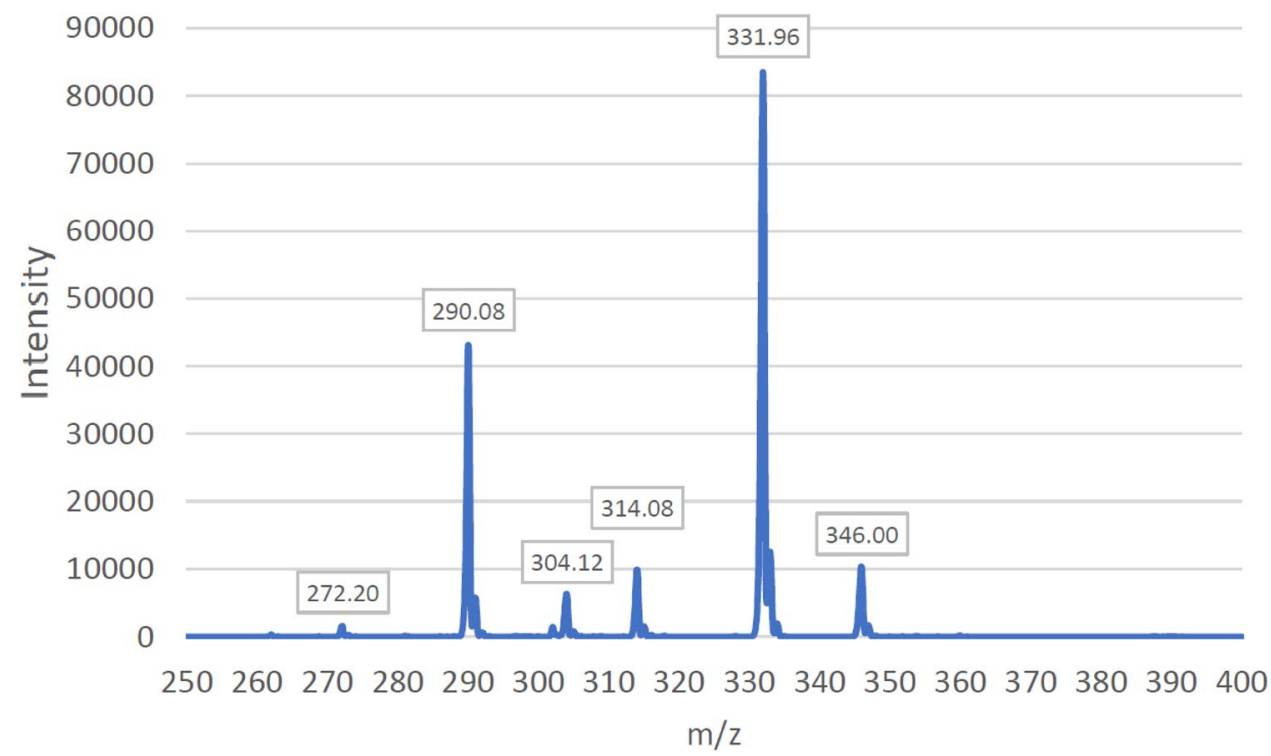




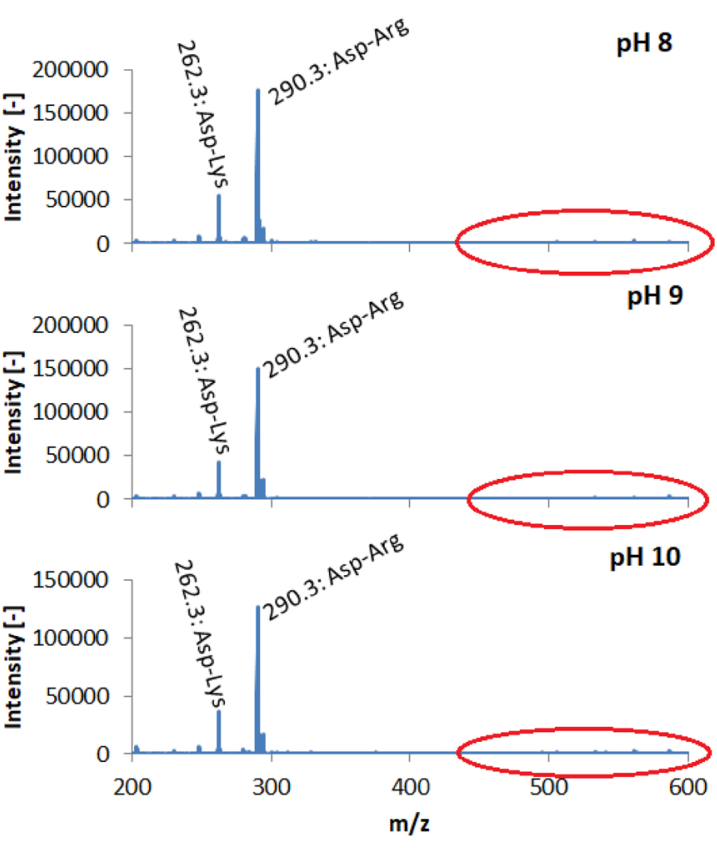

Fig. 11 MS analysis of the reaction of CGP dipepties and pyridoxal phosphate (PLP) at different $\mathrm{pH}$. Left: overview; right: enlargement of the relevant range which is indicated in red. The change in mass from $m / z=290.3$ to 518.4 for the Asp-Arg dipeptide and from $\mathrm{m} / z$

setups, since the change in mass is consistent with the acetylation of the known dipeptides.

The calculated conversion rates for the acetylation of CGP dipeptides were $>99 \%$ for Asp-Lys; $36.6 \%$ of the AspLys was acetylated once, while $63.4 \%$ were acetylated twice. $66 \%$ of Asp-Arg was acetylated. If the unknown signal at $\mathrm{m} / \mathrm{z}=314.08$ is caused by acetylation of the unknown signal at $m / z=272.2$, the conversion rate is $89.2 \%$.

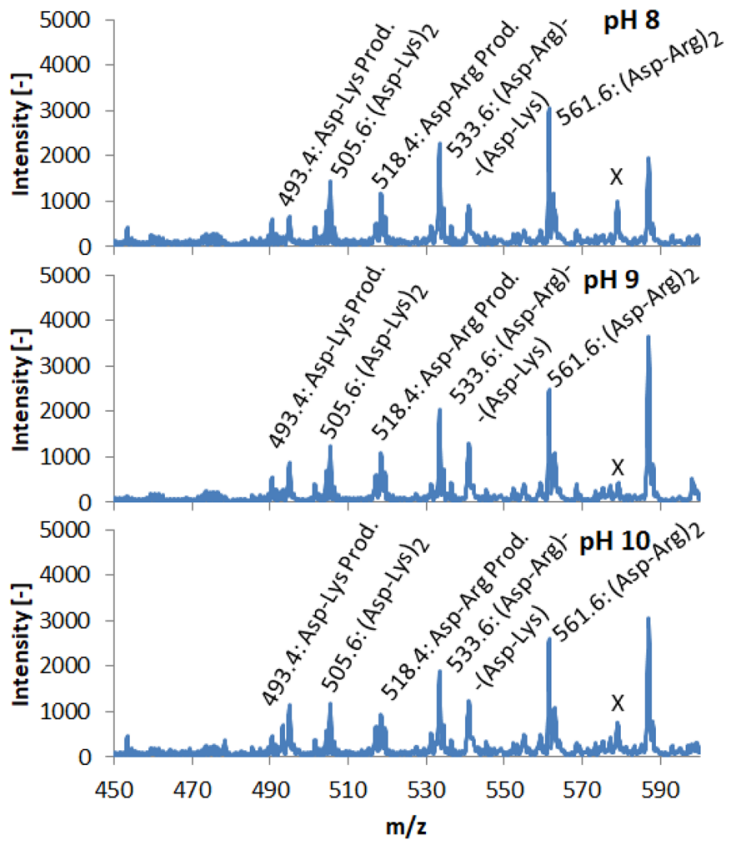

262.3 to 493.4 for the Asp-Lys dipeptide indicate the reaction with PLP. Other signals refer to tetra-peptides made of combinations of Asp-Arg and Asp-Lys. X may be an adduct of the asparate-arginine tetra-peptide and water with the $m / z=579.6$

\subsection{Reaction with Pyridoxal Phosphate}

MS spectra of the reaction with previously digested cyanophycin revealed a few peaks in the relevant mass range, even though they exhibited only very low intensities (see Fig. 11). The signal at $\mathrm{m} / \mathrm{z}=493.4$ corresponds with the expected mass for the modified Asp-Lys and the $m / z=518.4$ matches with the expected mass of modified Asp-Arg. In addition, the signal $\mathrm{m} / \mathrm{z}=491.4$ may be identified with the reaction product containing the non-reduced
Fig. 12 MS analysis of the reaction of a CGP dipeptide mixture with diacetyl. The mass change of the Asp-Arg dipeptide from $m / z=290.17$ to 376.00 corresponds to the addition of one diacetyl; the signal at $m / z=444.08$ to the addition of two molecules

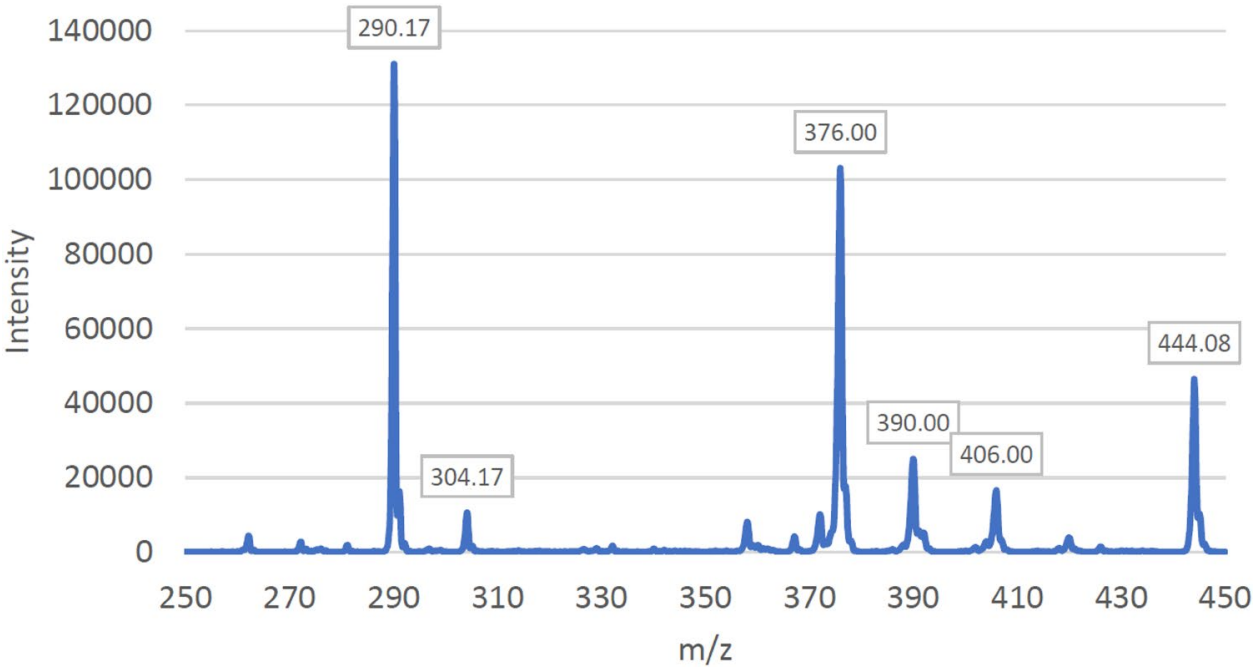


Schiff base. Since the fragmentation product yield was not high enough for verification, this peak is neglected in further calculations. Additional signals were identified as cyanophycin tetra-peptides resulting from incomplete digestion, $m / z=505.6$ for two Asp-Lys dipeptides, $m / z=533.6$ for a combination of one Asp-Lys and one Asp-Arg and $m / z=561.6$ for two Asp-Arg. The reaction showed no noticeable difference depending on $\mathrm{pH}$. About $2.2 \%$ of Asp-Lys and $<1 \%$ of Asp-Arg exhibited the expected mass of the reaction products.

Performing the same reaction for undigested cyanophycin, allowed for the usage of NaBH4 for stabilization of the resulting Schiff base formed by this reaction, however, there were no significant changes in MS analysis. Only the ratio of the signals at $m / z=493.4-491.4$ increased in regard to the already digested cyanophycin. This can be explained by the addition of sodium borohydride which reduces the formed Schiff base from a double bond to a single bond. A conversion rate of about $10 \%$ for the lysine dipeptide and $1-2 \%$ for the arginine dipeptide was determined, again with no clear correlation regarding the $\mathrm{pH}$.

\subsection{Diacetyl}

The MS analysis of cyanophycin incubated with diacetyl exhibits a dominant signal at $m / z=444.08$, which corresponds to the reaction of the Asp-Arg dipeptide with two molecules of diacetyl. If water is cleaved of this molecule $(m / z-18)$, the structure will lead to a signal at approx. $m / z=444.08$. Based on this, the conversion rate of Asp$\operatorname{Arg}(m / z=290.17)$ to the signal at $m / z=444.08$ was determined at $79.7 \%$. There was no measurable product of the Asp-Lys dipeptide at $m / z=262.17$.

Performing the reaction with already digested CGP shows a dominant signal at $m / z=376.00$ which refers to one of the possible reaction products of Asp-Arg reacting with a single diacetyl (Fig. 12). The signal at $m / z=390.00$ may indicate a similar modification but of the structure causing the signal at $m / z=304.17$, which was already measured in other dipeptide reaction setups and is of unknown origin. The signal at $m / z=444.08$ was already present in the reaction with CGP and refers to the Asp-Arg dipeptide reacting with two molecules of diacetyl. The signal at $m / z=406$ is unexpected. On the basis of the three peaks at $m / z=290.17,376.00$, and 444.08 and their predicted structures, the fraction of unmodified Asp-Arg to Asp-Arg reacted with one molecule of diacetyl $(\mathrm{m} / \mathrm{z}=376.00)$ was $36.7 \%$. The fraction of AspArg reacted with two molecules of diacetyl $(\mathrm{m} / \mathrm{z}=444.08)$ was $16.6 \%$. Again, no modification of Asp-Lys was detected.

Reactions performed with the generated diacetyl trimer exhibited a large variety of signals, none of which matched with predicted masses and/or signals detected in the reactions with regular diacetyl.

\section{Discussion}

\subsection{Cyanates}

The MS analysis of reaction products of cyanophycin with MITC demonstrates that a modification of CGP is possible and enhanced at high $\mathrm{pH}$ values. For the previously digested cyanophycin, a conversion of around $70 \%$ for the Asp-Lys dipeptide and around 95\% for the Asp-Arg was calculated. Hereby, the Asp-Lys dipeptide shows two possible modifications by single or double addition of MITC, whereas the Asp-Arg dipeptide shows one product. This pattern is explained by the fact that the Asp-Lys dipeptide yielded two amino moieties, the $\varepsilon$-group of the lysine residue and the $\alpha$-group of the aspartate, which is accessible in digested CGP, contrary to the undigested polymer. Asp-Arg possesses three amino groups, if the guanidine moiety of arginine is also taken into account, which can be modified. Therefore, three possible products should be found. However, due to the mesomeric stabilization of the guanidine residue, this group is not accessible to an addition reaction; therefore, only one product can be detected. This argumentation is supported by control reactions, which show a similar reaction behavior, as well as the reaction with undigested cyanophycin. Despite the solubility problem, a conversion rate of about $50 \%$ of Asp-Lys and 3\% for Asp-Arg was found. Thus, almost no modified Asp-Arg product was discovered. Also the amount of double modified Asp-Lys dipeptides was significantly decreased. The presence of low amounts of modified AspArg and double modified Asp-Lys dipeptides is explainable by the fact that not all cyanates could be removed after the reaction. The remaining cyanate traces may react with the dipeptides which are resulting from the digestion for analytic purposes. Also, the presence of dipeptides from the cyanophycin isolation or natural degradation process could not be excluded. Since performed control reaction with ornithine was promising, the reaction should also be done with ornithine-rich cyanophycin. By the introduction of an easily modifiable sulfur into cyanophycin upon reaction with MITC, the basis for additional linkage opportunity is built (Baslé et al. 2010). The solubility problem of the undigested CGP may be improved by varying the protocols resulting in a higher yield. The attempts to modify cyanophycin and cyanophycin dipeptides with ATIC and GITC were not successful. The control reaction with free lysine displayed a high conversion rate of approximately 55\% (ammonium isothiocyanate) and $>99 \%$ (guanidine isothiocyanate); however, the products were not corresponding to the expected masses and could thus not be identified. These other cyanate were used, due to their similar structure to MITC, but more promising may be the reaction with KNCO as described by Weisgraber et al. (1978). 


\subsection{Formaldehyde}

The cleavage products of cyanophycin obtained by incubation with CphE showed nearly no reaction (expected hydroxymethylation) at the Asp-Arg dipeptides, while $84.4 \%$ of the Asp-Lys dipeptides were methylated. The reaction setup with dipeptides (Fig. 8) showed completely different results. There was no methylation of Asp-Lys dipeptides measurable based on the done interpretation of the peaks, while $15.4 \%$ of the Asp-Arg dipeptides where both methylated and hydroxymethylated and an additional $11.8 \%$ was either methylated (5.4\%) or hydroxymethylated (6.4\%). One explanation for the absence of a methylated Asp-Lys is that a di-methylated Asp-Lys dipeptide may cause a signal at $m / z=290.2$, so unmodified Asp-Arg dipeptides and di-methylated Asp-Lys dipeptides may mask each other in the MS analysis. Due to this fact, all done measurements should be interpreted with care, especially the calculated conversion rates. However, the reaction, in general, concerning the conditions and buffers is a viable method.

\subsection{Acetic Anhydride}

Using undigested CGP as substrate, the acetylated Asp-Lys dipeptides showed a remarkable yield of $78.5 \%$ conversion rate. $4.7 \%$ of the Asp-Arg dipeptides were acetylated once without any other modification, but there occurred several other signals with substantial intensities which likely refer to acetylated dipeptides potentially with other modifications in common (Fig. 9). There are too many unknown peaks to include them for reasonable calculations of conversion rates. The interpretation of these signals is also difficult. Due to the controls that were done along with the reaction setup, it can be excluded that these signals originate from contaminations with other amino acids in the CGP or from other sources, they have to be modified Arg-Asp or Arg-Lys dipeptides. However, there are a couple of patterns noticeable within the detected signals. There is a recurring difference of $m / z=14$, applying to the peaks at $m / z=290.08$ and 304.08, to the peaks at $m / z=312.08$ and 326.08 , to the peaks at $m / z=334.17$ and 348.08 and also to the peaks at $\mathrm{m} / \mathrm{z}=356.08$ and 370.08. A difference of $14 \mathrm{~m} / \mathrm{z}$ might be caused by methylation as seen in the reaction with formaldehyde. A difference of $m / z=44$ can be seen between the peaks at $\mathrm{m} / z=290.08$ and 334.17 , the peaks at $\mathrm{m} / z=304.08$ and 348.08, the peaks at $m / z=312.08$ and 356.08, and also to the peaks at $m / z=326.08$ and 370.08 . Finally, there is also a pattern of a difference of $22 \mathrm{~m} / \mathrm{z}$ recognizable for the peaks at $m / z=290.08$ and 312.08, at $m / z=304.08$ and 326.08, at $m / z=312.08$ and 334.17, at $m / z=326.08$ and 348.08, at $\mathrm{m} / \mathrm{z}=334.17$ and 356.08 and for the peaks at $\mathrm{m} / \mathrm{z}=356.08$ and 370.08. A possible reason for both of these patterns could be the NaOAc buffer used in the reaction. The addition of sodium could result in a shift of $m / z=22$, while the acetate could contribute a carboxyl group for a change of $m / z=44$. However, this is unlikely since the identically treated dipeptides do not exhibit this change and they did not show up in control reactions with free amino acids. Therefore, further studies and experiments are required to identify the identity of these reaction products.

The measurement of the reactions performed on dipeptides showed notably better results. All Asp-Lys dipeptides where at least acetylated once, $63.4 \%$ of them were acetylated twice, $66 \%$ of the Asp-Arg dipeptides were acetylated and the structure causing the signal at $m / z=272.2$ was acetylated with a remarkable yield of $89.2 \%$. The signal at $\mathrm{m} / \mathrm{z}=272.2$ is puzzling, since the signal was too weak for MS-MS fragmentation to give analyzable results. The difference in mass of $m / z=18$ regarding the Asp-Arg dipeptide $(\mathrm{m} / \mathrm{z}=290.08)$ might suggest the loss of one molecule of $\mathrm{H}_{2} \mathrm{O}$ during the reaction or by other means.

\subsection{Pyridoxal Phosphate}

The achieved result for the reaction of the polymer with pyridoxal phosphate suggests that cyanophycin can be modified by the active form of vitamin B6. The maximal reached conversion rate for the Asp-Lys dipeptide of $10 \%$ was observed at a $\mathrm{pH}$ value of 9 and the use of sodium borohydride on undigested cyanophycin. It is worth to mention that the use of sodium borohydride increases also the yield, which may be reasoned by the reduction of the Schiff base and thus stabilizing the bond between the amino acid and the pyridoxal phosphate, due to the removal of the double bond. Also experiments studying the dependency of the conversion rate on the sodium borohydride concentration were interesting. The obtained data suggested also that a reaction with arginine is not preferred. Due to the mesomeric stabilization, the guanidine group cannot be attacked by pyridoxal phosphate. Since no significant products of the reaction with arginine were detected, this could indicate the specificity of the reaction towards non- $\alpha$-amino moieties. This should be substantiated by measurements of the reaction with free lysine in higher $\mathrm{m} / \mathrm{z}$ ranges, to detect a possible formation of products with two additions of pyridoxal phosphate. Based on the modification of cyanophycin by pyridoxal phosphate, the dephosphorylation of the product by acid phosphatase (Bingham et al. 1976; Masuda et al. 2005) is a promising next step for modifications.

\subsection{Diacetyl}

In comparison to the methylation or acetylation, the experiments with diacetyl had totally different preconditions. 
There is little literature available about this reaction, since it is rarely used. The reaction with diacetyl gave remarkable yields of $79.7 \%$ for the peak at $m / z=444$ in the reaction setup with $\mathrm{CphE}$ cleavage. The reaction with CGP gave no compound with a measurable signal at $m / z=376$, while the reaction with dipeptides exhibited a dominant signal. An explanation for this is that the peak at $m / z=376$ is caused by a modification which can be done on dipeptides but not on cyanophycin, so probably the amino group of aspartic acid is targeted and not the arginine. Due to these results, the structure causing the peak at $m / z=376$ is likely not a modification on arginine as desired. In general, the reactions with diacetyl offer an interesting basis for further experiments, since there is still not much knowledge about these reactions in comparison to established reactions like methylation or acetylation. Reactions performed with the generated diacetyl trimer, while exhibiting various reaction products were difficult to interpret and would require excessive additional experiments, exceeding the aim of this study.

In summary, the tested reactions exhibited significant conversion rates for both arginine and lysine but were often dependent on the enzymatic digestion of the CGP into dipeptides, which except for the methylation of lysine residues, usually gave higher conversion rates than undigested CGP. Some of these modifications might have actual applications, e.g. the aforementioned use of acetylation to increase the permeability of certain drugs through the blood-brain barrier (Pardridge 2012) or conversion of N6-(pyridoxal phosphate)-lysine of the modified Asp-Lys dipeptide into regular pyridoxal (vitamin $\mathrm{B}_{6}$ ) by dephosphorylation (Masuda et al. 2005). Methylation, in addition to its importance as post-translational modification of arginine and lysine, also has interesting medical applications: Oligo-arginines are studied due to their capability in penetrating cell-walls and as part of herbicides and anti-malaria drugs (Sparr et al. 2013). Grogg et al. (2018) introduced modifications like $\mathrm{MeO}, \mathrm{Me}_{2} \mathrm{~N}$, or $\mathrm{Me}_{3} \mathrm{CO}$ to CGP-derived octapeptides, which were used due to their similarity to oligo-arginines and found significant differences in the cell-wall permeability and toxicity of the compounds, depending on the introduced modification. A different set of methylation or other modifications could expand on these results.

Other modifications could be used to further expand the toolbox for introducing novel modifications into the CGP. The introduction of a sulfur group using isothiocyanates opens up a new spectrum of reactions targeting this group instead of the functional groups available on the unmodified arginine or lysine. Acetylation (and in part methylation) can provide protecting groups to block certain groups from modification (Kociensky 2005) which could be desirable for reactions targeting both the arginine and lysine residues. Using undigested CGP acetylation and methylation primarily affected the lysine $(78.5 \%$ and $84.4 \%)$ with only minor changes to the arginine $(4.7 \%$ and $0.0 \%)$.

Overall, the investigated reactions confirmed the viability of chemical CGP modification, especially in comparison to enzymatic modifications.

\section{Compliance with Ethical Standards}

Conflict of interest On behalf of all authors, the corresponding author declares that there is no conflict of interest and no financial and/or personal relationship with a third party.

Open Access This article is distributed under the terms of the Creative Commons Attribution 4.0 International License (http://creativeco mmons.org/licenses/by/4.0/), which permits unrestricted use, distribution, and reproduction in any medium, provided you give appropriate credit to the original author(s) and the source, provide a link to the Creative Commons license, and indicate if changes were made.

\section{References}

Baslé E, Joubert N, Pucheault M (2010) Protein chemical modification on endogenous amino acids. Chem Biol 17:213-227

Bingham EW, Farrell HMJ, Dahl KJ (1976) Removal of phosphate groups from casein with potato acid phosphatase. Biochim Biophys Acta 429:448-460

Cresswell RM, Smith WRD, Wood HCS (1961) The structure of two trimers of biacetyl. J Chem Soc 0:4882-4885.

Fraenkel-Conrat H (1957) Methods for investigating the essential groups for enzyme activity. Methods Enzymol 4:247-269

Frommeyer M, Bergander K, Steinbüchel A (2014) Guanidination of soluble lysine-rich cyanophycin yields a homoarginine-containing polyamide. Appl Environ Microbiol 80:2381-2389

Greenhalgh R, Bannard RAB (1961) Guanidine compounds: IV. Acetylation of some alkyl-substituted guanidines with acetic anhydride and ethyl acetate. Can J Chem 39:1017-1029

Grogg M, Hilvert D, Ebert MO, Beck AK, Seebach D, Kurth F, Dittrich PS, Sparr C, Wittlin S, Rottmann M, Mäser P (2018) Cell penetration, herbicidal activity, and in-vivo-T'toxicity of oligo-arginine derivatives and of novel guanidinium-rich compounds derived from the biopolymer cyanophycin. Helv Chim Acta 101:10

Harden A, Norris D (1911) The diacetyl reaction for proteins. J Physiol 42:332-336

Herriott RM (1947) Reaction of native proteins with chemical reagents. Adv Protein Chem 3:169-225

Hubert E, Ojeda A, Reyes A, Slebe JC (1986) Potassium activation and its relationship to a highly reactive cysteine residue in fructose 1,6-bisphosphatase. Arch Biochem Biophys 250:336-344

Jaisson S, Lorimier S, Ricard-Blum S, Sockalingum GD, DelevalléeForte C, Kegelaer G, Manfait M, Garnotel R, Gillery P (2006) Impact of carbamylation on type I collagen conformational structure and its ability to activate human polymorphonuclear neutrophils. Chem Biol 13:149-159

Jentoft N, Dearborn DG (1979) Labeling of proteins by reductive methylation using sodium cyanoborhydride. J Biol Chem 254:4359-4365

Kocienski PJ (2005) Protecting Groups, 3rd edn. Georg Thieme Verlag, Stuttgart

Kroll J, Klinter S, Steinbüchel A (2011) A novel plasmid addiction system for large-scale production of cyanophycin in Escherichia 
coli using mineral salts medium. Appl Microbiol Biotechnol 89:593-604

Lundblad RL (1994) Techniques in protein modification. CRC Press, Boca Raton

Masuda T, Ide N, Kitabatake N (2005) Effects of chemical modification of lysine residues on the sweetness of lysozyme. Chem Senses 30:253-264

Mathews JM, Watson SL, Snyder RW, Burgess JP, Morgan DL (2010) Reaction of the butter flavorant diacetyl (2,3-butanedione) with $\mathrm{N}$-acetylarginine: a model for epitope formation with pulmonary proteins in the etiology of obliterative bronchiolitis. J Agric Food Chem 58:12761-12768

Means GE (1977) Reductive alkylation of amino groups. Methods Enzymol 47:469-478

Means GE, Feeney RE (1968) Reductive alkylation of amino groups in proteins. Biochemistry 7:2192-2201

Miles EW (1986) In: Dolphin D, Poulson R, Avramovic O (eds) Vitamin B6 pyridoxal phosphate, chemical, biochemical, and medical aspects Part B. Wiley-Interscience, New York, pp 253-310

Pal J, Bera S, Ghosh SK (1999) Acetylation of $\alpha$-crystallin with $N$-acetylimidazole and its influence upon the native aggregate and subunit reassembly. Curr Eye Res 19:358-367

Pardridge W (2012) Drug transport across the blood-brain barrier. J Cereb Blood Flow Metab 32:1959-1972

Riordan JF, Wacker WEC, Vallee BL (1965) N-Acetylimidazole-a reagent for determination of free tyrosyl residues of proteins. Biochemistry 4:1758-1765

Sallam A, Steinbüchel A (2010) Dipeptides in nutrition and therapy: cyanophycin-derived dipeptides as natural alternatives and their biotechnological production. Appl Microbiol Biotechnol 87:815-828

Sallam A, Kast A, Przyilla S, Meiswinkel T, Steinbüchel A (2009) Biotechnological process for production of $\beta$-dipeptides from cyanophycin on a technical scale and its optimization. Appl Environ Microbiol 75:29-38

Scherer HJ, Karthein R, Strieder S, Ruf HH (1992) Chemical modification of prostaglandin endoperoxide synthase by $N$-acetylimidazol effect on enzymic activities and EPR spectroscopic properties. Eur J Biochem 205:751-757

Shapiro S, Enser M, Pugh E, Horecker B (1968) The effect of pyridoxal phosphate on rabbit muscle aldolase. Arch Biochem Biophys 128:554-562

Sparr C, Purkayastha N, Kolesinska B, Gengenbacher M, Amulic B, Matuschewski K, Seebach D, Kamena F (2013) Improved efficacy of fosmidomycin against Plasmodium and Mycobacterium species by combination with the cell-penetrating peptide octaarginine. Antimicrob Agents Chemother 57:4689-4698

Stark GR (1972) Modification of proteins with cyanate. Methods Enzymol 25:579-584

Steinle A, Bergander K, Steinbüchel A (2009) Metabolic engineering of Saccharomyces cerevisiae for production of novel cyanophycins with an extended range of constituent amino acids. Appl Environ Microbiol 75:3437-3446

Sun S, Zhou JY, Yang W, Zhang H (2014) Inhibition of protein carbamylation in urea solution using ammonium-containing buffers. Anal Biochem 446:76-81

Trézl L, Hullán L, Jászay ZM, Szarvas T, Petneházy I, Szende B, Bocsi J, Takáts Z, Vékey K, Tóke L (2003) Antagonistic reactions of arginine and lysine against formaldehyde and their relation to cell proliferation, apoptosis, folate cycle and photosynthesis. Mol Cell Biochem 244:167-176

Wagner GR, Payne MR (2013) Widespread and enzyme-independent $N^{\prime}$-acetylation and $N^{\prime}$-succinylation of proteins in the chemical conditions of the mitochondrial matrix. J Biol Chem 288:29036-29045

Weinert B, Iesmantavicius V, Wagner S, Schölz C, Gummesson B, Beli P, Nyström T, Choudhary C (2013) Acetyl-phosphate is a critical determinant of lysine acetylation in E. coli. Mol Cell 51:265-272

Weisgraber KH, Innerarity TL, Mahley RW (1978) Role of lysine residues of plasma lipoproteins in high affinity binding to cell surface receptors on human fibroblasts. J Biol Chem 253:9053-9062

Wiefel L, Steinbüchel A (2014) Solubility behavior of cyanophycin depending on lysine content. Appl Environm Microbiol 80:1091-1096

Wiefel L, Steinbüchel A (2016) Enzymatic modification of soluble cyanophycin using the type II peptidyl arginine deiminase from Oryctolagus cuniculus. Macromol Biosci 16:1064-1071

Wiefel L, Bröker A, Steinbüchel A (2011) Synthesis of a citrulline-rich cyanophycin by use of Pseudomonas putida ATCC 4359. Appl Microbiol Biotechnol 90:1755-1762

Yankeelov JJ (1970) Modification of arginine in proteins by oligomers of 2,3-butanedione. Biochemistry 9:2433-2439

Yankeelov JJ, Mitchell C, Crawford T (1968) A simple trimerization of 2,3-butanedione yielding a selective reagent for the modification of arginine in proteins. J Am Chem Soc 90:1664-1666

Zhang F, Gao J, Weng J, Tan C, Ruan K, Xu C, Jiang D (2005) Structural and functional differentiation of three groups of tyrosine residues by acetylation of $\mathrm{N}$-acetylimidazole in manganese stabilizing protein. Biochemistry 44:719-725 